\title{
Los efectos adversos de la luz artificial por la noche
}

\author{
Natalí N. Guerrero-Vargas, \\ Manuel Ángeles-Castellanos y \\ Carolina Escobar Briones
}

\section{Resumen}

Nuestro cuerpo tiene un reloj biológico y todos los tejidos son osciladores que siguen al ciclo diario de luzoscuridad para poder cambiar la intensidad de la conducta y nuestras funciones fisiológicas y de esta forma hacernos más eficientes según si estamos despiertos o dormidos. Nuestro reloj biológico reconoce cuando está oscuro y cuando hay luz y lo interpreta como día y noche. Con el uso de la luz eléctrica, que comenzó alrededor del siglo pasado, este sistema temporal ha sido alterada. Los científicos han comenzado a señalar que la exposición a la luz por la noche confunde al reloj biológico lo cual puede tener consecuencias sociales, ecológicas, conductuales y para la salud. Las personas que se exponen a la luz eléctrica por largas horas durante la noche son las más afectadas, particularmente los trabajadores nocturnos. Sin embargo, cambios en el estilo de vida han llevado a que jóvenes y niños se expongan desde edades tempranas a este fenómeno conocido como "contaminación lumínica". En este texto pretendemos exhibir las evidencias clínicas y experimentales que indican que la luz artificial por la noche es un factor adverso, promotor de alteraciones en el sistema circadiano, en la fisiología y, por lo tanto, la contaminación lumínica es un factor de riesgo para la salud pública.

Palabras clave: ritmos circadianos, contaminación lumínica, depresión, alteraciones circadianas, síndrome metabólico, cáncer.

\section{The negative effects of artificial light at night Abstract}

\begin{abstract}
Our body has a biological clock and all tissues are oscillators that follow the daily light-dark cycle in order to change the intensity of behavior and physiological functions and make us more efficient according to whether we are awake or asleep. The invention and use of electric lights, which began around the last century, have affected this temporal organization. Light exposure at night has social, ecological, behavioral and health consequences that just now begin to be apparent. People with nocturnal habits are mostly exposed to light at night, and among them night workers are at risk. Due to changes in life style, young people, including children, are now individuals exposed to this factor that affects their physiology. The excess of light at night, also called "light pollution", is the cause of diverse health problems. The aim of this paper is to present evidence from clinical studies and experimental models that points out the deleterious effects of light at night as a factor that affects the circadian system, physiological systems and behavior leading to disease. Here we offer evidence that light at night is a risk factor for public health.
\end{abstract}

Key words: circadian rhythms, light pollution, depression, circadian disruption, metabolic syndrome, cancer. 


\section{Natalí N. Guerrero-Vargas}

Profesora de tiempo completo en el Departamento de Anatomía de la Facultad de Medicina de la Universidad Nacional Autónoma de México (UNAM) donde es co-responsable del laboratorio de Ritmos biológicos y Metabolismo. Pertenece al Sistema Nacional de Investigadores (SNI nivel I). Es Bióloga por la Facultad de Ciencias, UNAM (2009) y doctora en Ciencias, UNAM (2015). Participa en actividades docentes en la UNAM y en la Universidad Panamericana. Ha publicado diversos artículos en revistas internacionales y ha presentado su trabajo de investigación en congresos nacionales e internacionales. Su línea de investigación se enfoca en entender como diversos procesos que alteran los ritmos circadianos promueven el desarrollo de enfermedades.

\section{Manuel Ángeles-Castellanos}

Profesor de tiempo completo y Jefe del Departamento de Anatomía de la Facultad de Medicina de la Universidad Nacional Autónoma de México (UNAM), es responsable del laboratorio de Cronobiología. Pertenece al Sistema Nacional de Investigadores (SNI nivel I). Es Médico Cirujano. Egresado de la Facultad de Medicina y Cirugía de la Universidad Benito Juárez de Oaxaca (1991-1997) y doctor en Ciencias Biomédicas (2005) por la UNAM. Es miembro regular de la academia nacional de medicina de México. Sus líneas de investigación: contribución del alimento palatable (chocolate) en los mecanismos centrales de re-sincronización del sistema circadiano, después de un avance de fase de seis horas.

\section{Carolina Escobar Briones}

escocarolina@gmail.com

Profesora de tiempo completo en el Departamento de Anatomía de la Facultad de Medicina UNAM, donde actualmente funge como coordinadora de investigación y es responsable del laboratorio de Ritmos biológicos y metabolismo. Es profesora de asignatura en la Facultad de Psicología de la misma universidad. Pertenece al Sistema Nacional de Investigadores (SNI nivel III). Cuenta con más de 140 publicaciones. Fungió como editora en dos números de la revista Biological Rhythms Research (1998 y 2005) y para un número especial la revista Ciencia (2008) de la Academia Mexicana de Ciencias titulado "Ritmos biológicos". Desde el 2013 es miembro del Comité editorial de la revista Biological Rhythms Research. Sus líneas de investigación: el alimento como factor de desincronización circadiano, modelos de desincronización circadiana para el estudio de la obesidad, la sincronización por alimento como desencadenante de adicción. 


\section{Introducción: el sistema circadiano y la luz como señal de tiempo}

La sociedad moderna ha adoptado un estilo de vida que violenta la organización temporal de nuestra conducta y fisiología. Las condiciones ambientales del día y la noche que se requieren para ajustar las diversas funciones fisiológicas en nuestro organismo se respetan poco y esto afecta la eficiencia con la que nos adaptamos y respondemos a los cambios del medio ambiente externo.

Nuestra adaptación al ciclo luz-oscuridad depende del sistema circadiano, que tiene la función de monitorear señales temporales externas y transmitirlas al resto del cuerpo. El sistema circadiano consiste en una especie de reloj principal (Buijs y Kalsbeek, 2001), localizado en el cerebro llamado núcleo supraquiasmático (NSQ) y por su interacción con tejidos del cuerpo con capacidad de

Figura 1. Oscilar con ciclos de 24 horas, conocidos como osciladores periféricos (Buijs, van Esquema del sistema Eden, Goncharuk y Kalsbeek, 2003).

circadiano.
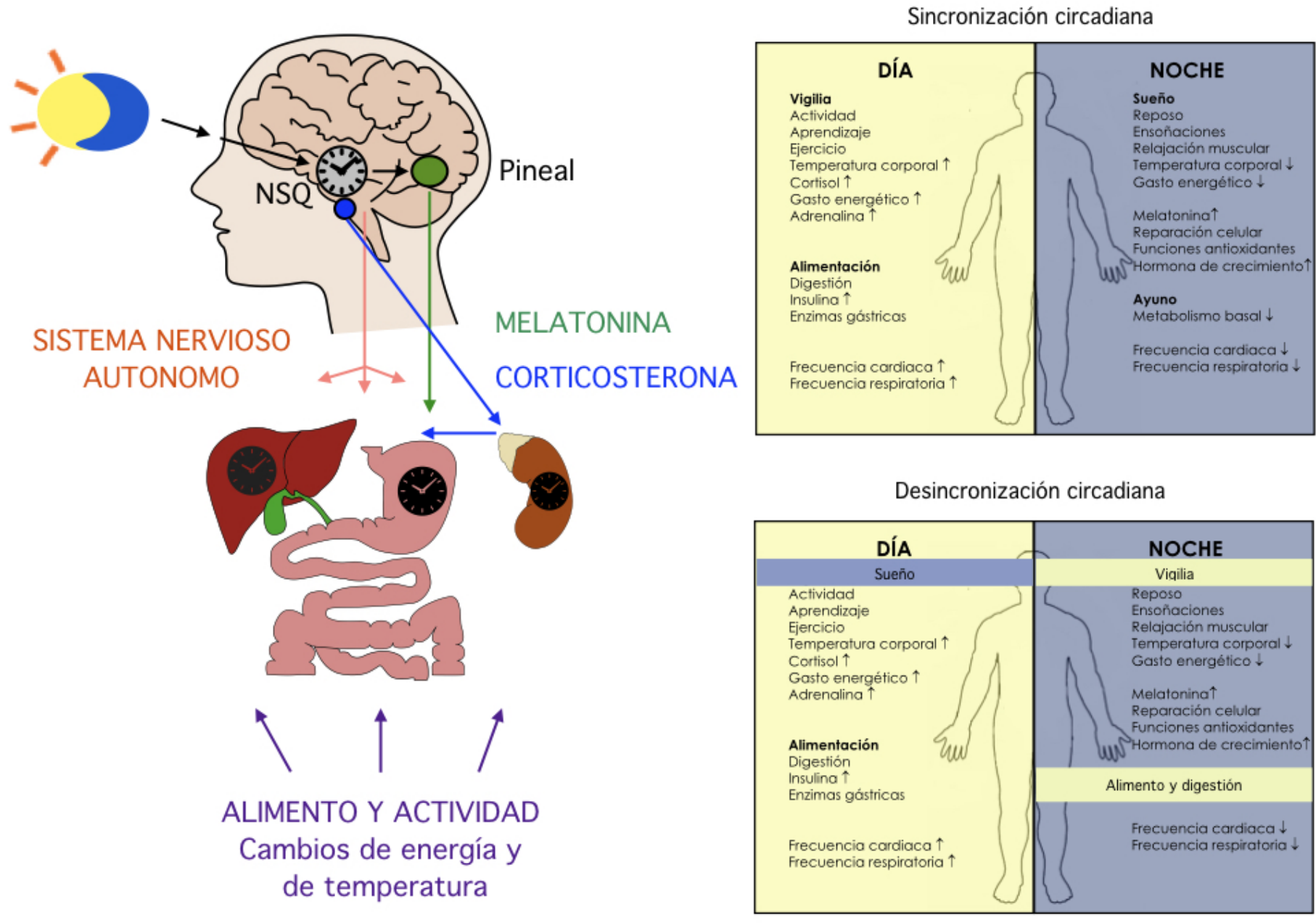

Desincronización circadiana

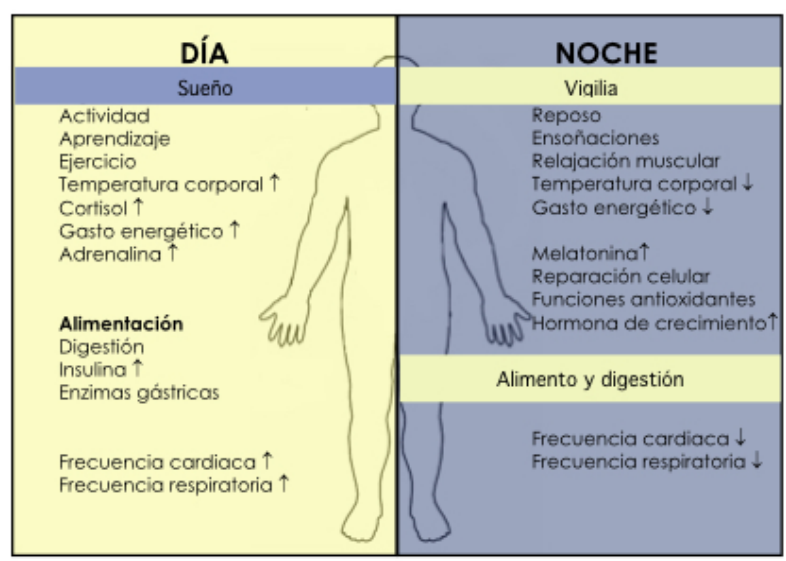


1. Las células ganglionares son un tipo de neurona localizada en la retina del ojo.
Este reloj biológico transmite ritmos de 24 horas a todos los tejidos por medio de señales neurales y señales hormonales (melatonina y corticosterona). Por este proceso se logra que la conducta y las funciones internas respondan de forma coordinada dependiendo de la hora del día, lo cual se conoce como sincronización circadiana. Cuando el individuo realiza actividades en conflicto con las señales del reloj biológico, por ejemplo, encender la luz cuando debería de estar oscuro, se provoca una desincronización circadiana, lo cual lleva a que muchas funciones se realicen con una intensidad inadecuada para los requerimientos del ambiente (ver figura 1).

La referencia temporal más potente que rige al sistema circadiano es la alternancia del ciclo luz-oscuridad y son las células ${ }^{1}$ de la retina las que le comunican al reloj biológico el estado de luminosidad correspondiente al día y la noche. La señal de luz activa a las neuronas del NSQ y activa genes cíclicos conocidos como genes reloj, que miden el tiempo a nivel celular (Golombek y Rosenstein, 2010). Otros eventos externos cíclicos que también afectan al sistema circadiano son los cambios de temperatura, sonidos sociales, el consumo de alimento, etcétera (Danilenko, Cajochen y Wirz-Justice, 2003; Klerman et al., 1998).

EI NSQ impone ciclos de actividad a otras áreas cerebrales para darle tiempo a la conducta y para transmitir señales de tiempo al resto del organismo a través de proyecciones del sistema nervioso autónomo y por medio la producción de la hormona melatonina -secretada por la glándula pineal-y la corticosterona - producida por el eje hipotálamo-hipófisis-adrenal(Kalsbeek et al., 2006). Los tejidos del cuerpo reconocen estas señales neuronales y hormonales y según su intensidad emiten respuestas convenientes para la fase del día o la noche (como se puede ver en la figura 1). Para la adecuada sincronización del sistema circadiano se requiere que las señales de luz-oscuridad que recibe el reloj biológico concuerden con las señales que recibe del resto del cuerpo sobre las actividades que realiza. Cuando estas señales están descoordinadas confunden al sistema circadiano y lo llevan a perder el orden temporal, lo cual repercute en la conducta y en la fisiología del individuo.

Hoy en día, nuestras casas, lugares de trabajo y calles están iluminadas por luz artificial brillante durante el día y durante la noche. La exposición a la luz artificial de noche provoca la pérdida de una alternancia del ciclo día-noche y representa un factor de riesgo para la coordinación del reloj biológico. El mal funcionamiento del sistema circadiano a largo plazo desencadena padecimientos crónicos, entre 


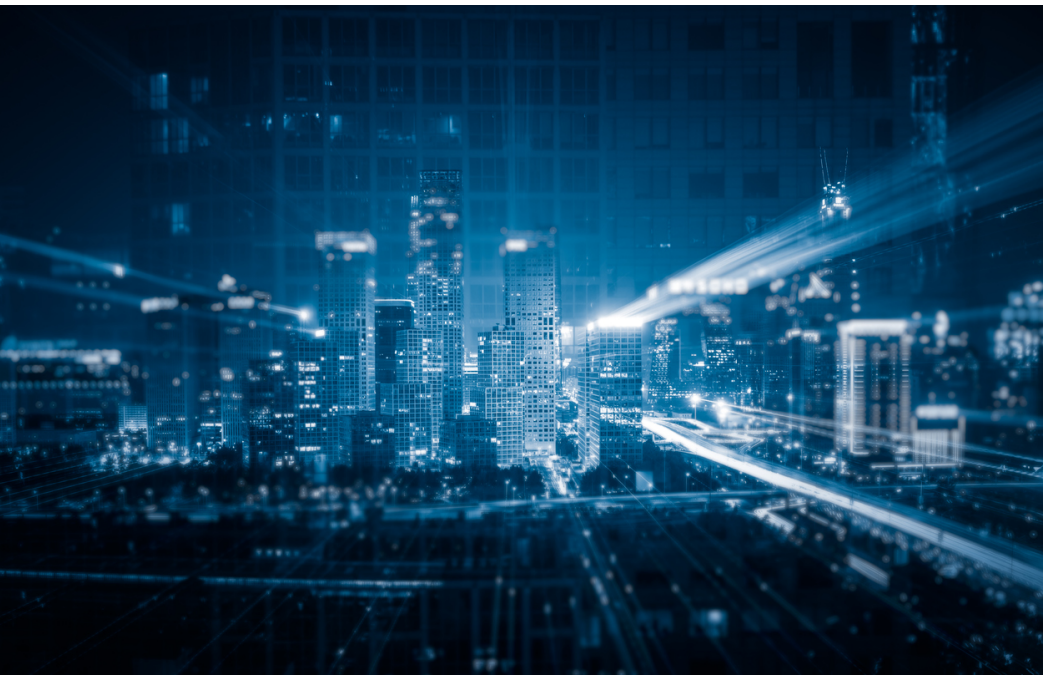

ellos enfermedades metabólicas, cáncer y alteraciones del estado de ánimo (Escobar et al., 2011).

Son varias las condiciones que pueden desencadenar alteraciones circadianas, entre ellas, la más común es el trabajo nocturno, también el síndrome de jet-lag resultante de los viajes trasmeridionales, el alimento y los estímulos luminosos durante la noche. Existe amplia literatura que aborda la relación de la alteración circadiana principalmente asociada con el trabajo nocturno y el jet-lag. Sin embargo, los efectos de la exposición a la luz artificial durante la noche sobre el sistema circadiano y la salud apenas comienzan a ser estudiados.

Este artículo tiene como objetivo definir en qué consiste el problema de la contaminación lumínica asociada a la luz artificial por la noche. Presentaremos evidencias clínicas y experimentales que indican que la exposición a la luz por la noche es un factor adverso, promotor de alteraciones circadianas y de problemas de salud. También exploraremos los posibles mecanismos que asocian al sistema circadiano alterado por la exposición a luz nocturna con una mayor propensión a desarrollar enfermedades.

\section{El problema de la contaminación lumínica: el exceso de luz por la noche}

La luz es esencial para la vida en la Tierra, es una fuente de energía, de calor, y mantiene los niveles de oxígeno en la atmósfera debido al importante papel que juega en la fotosíntesis.

Por miles de años el hombre ha vivido en un ambiente donde la luz corresponde a la fase de actividad y la noche a la fase de descanso. A lo largo de la historia, la posibilidad de alumbrar la noche dependió de la flama tenue de una hoguera o de una antorcha y fue apenas hace poco más de 100 años que Tomás Alba Edison (1879) inventó la bombilla eléctrica y fue posible alumbrar la noche con una mayor intensidad. El siglo XX se caracterizó por la implementación de luz eléctrica en casas y sitios de trabajo, permitiendo extender las horas de trabajo y estudio a la noche (Chepesiuk, 2009), lo cual modificó también el estilo de vida. Actualmente en las ciudades y pueblos se abusa del uso de la luz por la noche, proceso que conocemos como "contaminación lumínica". El abuso de iluminación es tal, que se irradia a muchos kilómetros más allá de las ciudades, iluminando los campos, las montañas y la naturaleza en general. Esta luz nocturna afecta patrones de migración de algunas aves e insectos y en algunas 
Figura 2.

El teléfono celular es una fuente de luz nocturna, que irradia luz directamente a los ojos del individuo que los está empleando.

Foto: $\underline{\text { ladyslav Dukhin. }}$

especies altera los ritmos de reproducción (Navara y Nelson, 2007). La iluminación urbana también produce "invasión lumínica", que ocurre cuando la luz del alumbrado público irradia hacia el interior de las casas, ocasionando un nivel de luminosidad constante aun teniendo las luces apagadas (Falchi, Cinzano, Elvidge, Keith y Haim, 2011). Otra fuente de luz nocturna proviene de los aparatos electrónicos como pantallas de televisión, computadoras, teléfonos celulares y tabletas, que irradian luz directamente a los ojos del individuo que los está empleando (ver figura 2).

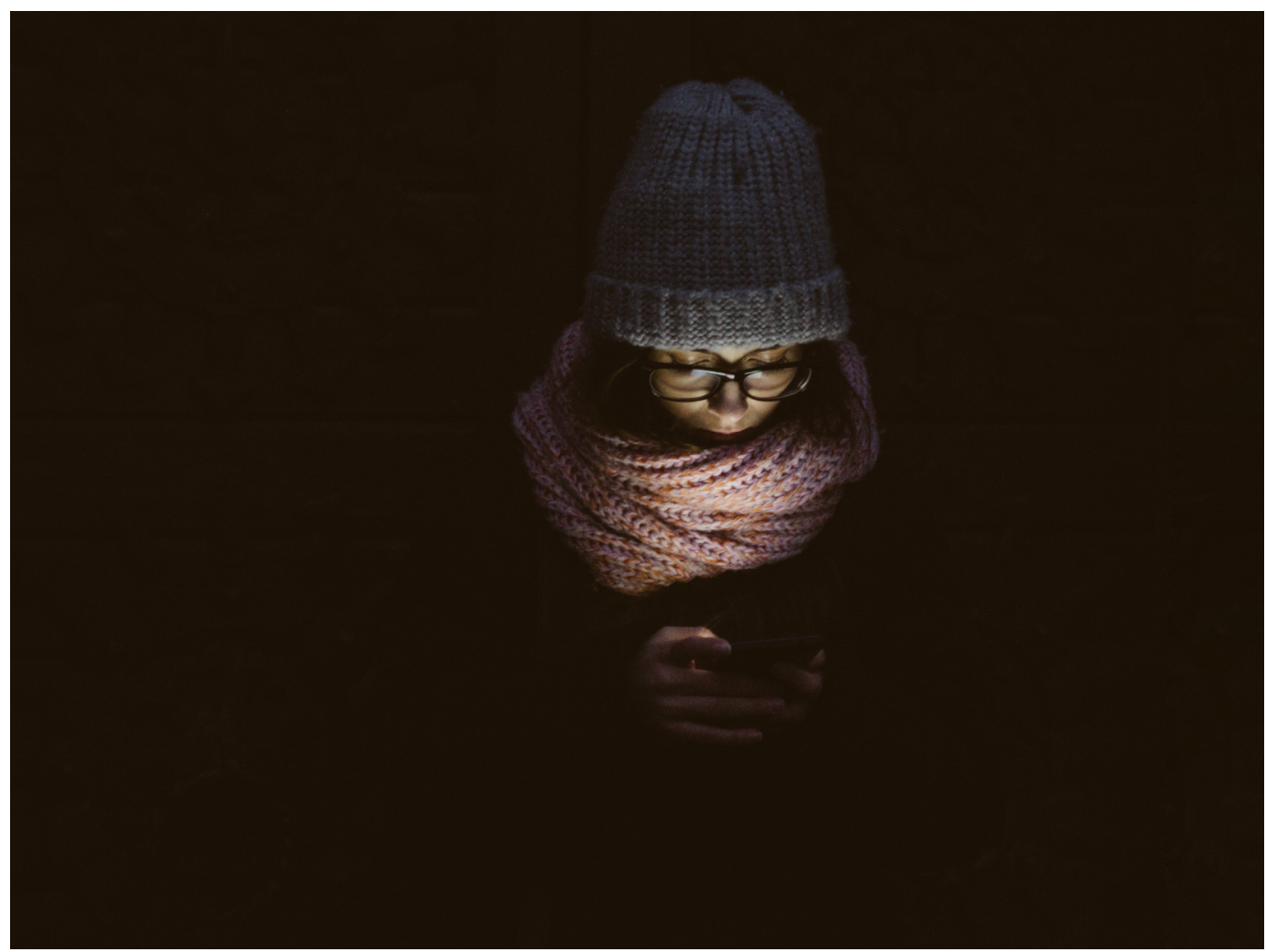

\section{¿Cuánto es mucha luz?}

En un día soleado, la iluminación puede llegar a alcanzar hasta los 100,000 lux², pero en un cuarto bien iluminado la intensidad de la luz alcanza los 1000 lux. Preferentemente en la recámara en donde vamos a dormir y con la luz apagada, la intensidad debiera ser menor a 10 lux.

Inicialmente los especialistas en ritmos circadianos consideraron como dañina una intensidad de luz de 7000 a 13000 lux debido a que alteraba al reloj biológico y con ello la expresión de los ritmos circadianos (Boivin, Duffy, Kronauer y Czeisler, 1996). Sin embargo, tomando como referencia el ritmo diario de melatonina, hormona que se secreta solamente por la noche y es inductora del sueño, se determinó que intensidades de hasta 0.2 lux de luz blanca inhiben inmediatamente 
su producción (Nathan, Burrows y Norman, 1999; Pauley, 2004). Al respecto se ha determinado que la longitud de onda de 440 a $460 \mathrm{~Hz}$ que corresponde a la luz azul, aún con intensidad baja de 1 lux, es suficiente para suprimir la secreción de melatonina (Stevens, Brainard, Blask, Lockley y Motta, 2013). Es importante resaltar que las pantallas de televisión, de computadoras y tabletas emiten principalmente longitudes de onda correspondientes al espectro azul (Zeitzer, Dijk, Kronauer, Brown y Czeisler, 2000). En fuentes de luz con un espectro más amplio de longitudes de onda (por ejemplo, lámparas del alumbrado público) una intensidad de 100 lux tiene el mismo efecto. Esta luz también modifica la actividad del NSQ el cual, como ya se describió, utiliza como referencia temporal la alternancia día-noche para sincronizar sus oscilaciones diarias y coordinar los ritmos circadianos del organismo. Actualmente en la mayoría de las casas durante la noche, las fuentes de luz emiten iluminación de hasta 1000 lux, intensidad suficiente para retrasar el inicio del sueño, causar alteraciones circadianas e inhibir la secreción de melatonina (Reiter, 2006).

\section{Consecuencias de la luz por la noche: estudios clínicos}

La luz por la noche promueve un estado de alerta conductual, que retrasa el inicio del sueño y promueve al desvelo. En días de trabajo o escuela estos desvelos tienen como consecuencia que se acorten las horas de descanso y ello ocasiona una privación de sueño crónica, que afecta el rendimiento escolar y laboral (van Cauter et al., 2007). Una preocupación actual es que en sujetos jóvenes, la exposición a luz artificial durante la noche (5 a 10 lux) afecta la calidad del sueño, aumenta la frecuencia de los despertares, la cantidad de sueño superficial y disminuye la cantidad de movimientos oculares rápidos (Cho et al., 2016). En un trabajo pionero de Lewy, Wehr, Goodwin, Newsome, y Markey (1980), se exploraron los efectos de la exposición a diferentes intensidades de luz por la noche en un rango de 0.03 hasta 9.5 lux y se observó que la exposición a todas las intensidades de luz provoca a corto plazo efectos adversos sobre los ritmos hormonales y de temperatura (Boivin et al.,1996). En un estudio más reciente se reportó que, en personas jóvenes la exposición a la luz por la noche, con una intensidad equivalente a la pantalla de una computadora, suprime la liberación de melatonina (Gooley et al., 2011).

Actualmente un 30\% de la población mundial trabaja por la noche (Rajaratnam y Arendt, 2001) y esta es la población más expuesta a la luz nocturna. En trabajadoras nocturnas se ha descrito una mayor propensión a desarrollar cáncer de mama (He, Anand, Ebell, Vena y Robb, 2015) y se ha determinado que el haber trabajado 15 años o más en turnos rotatorios o nocturnos incrementa hasta un 28\% el riesgo de padecer cáncer de pulmón en mujeres fumadoras (Schernhammer, Feskanich, Liang y Han, 2013). En enfermeras que trabajaron turnos nocturnos y estuvieron expuestas a la luz brillante mientras dormían, se 
demostró que los niveles de melatonina eran menores en comparación con enfermeras que trabajaron turnos diurnos (Grundy et al., 2009). Igualmente, entre los trabajadores nocturnos masculinos hay mayor propensión a padecer cáncer de próstata, además de presentar un mayor riesgo de eventos cardiovasculares con riesgo de infarto (Sigurdardottir et al., 2012). El trabajador nocturno también tiene mayor predisposición a la acumulación de adiposidad, sobrepeso y elevada predisposición a desarrollar enfermedades metabólicas incluyendo diabetes (Knutsson, 2003).

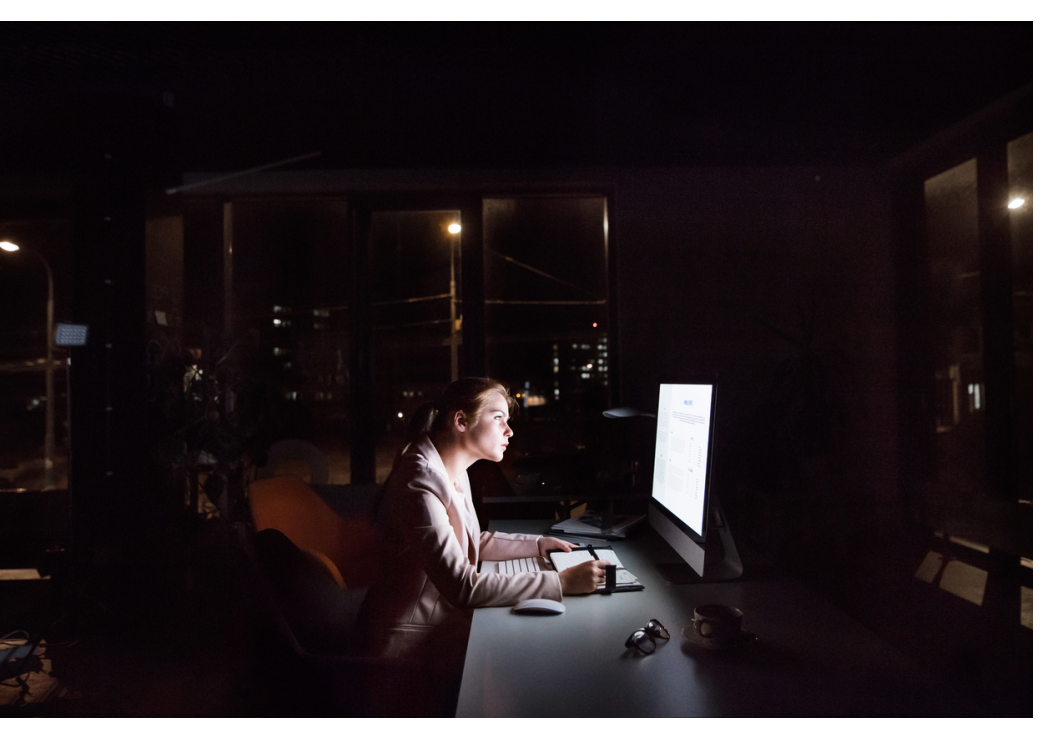

A nivel de la conducta, se ha reportado mayor propensión a cambios en el estado de ánimo, caracterizados por mayor irritabilidad, fatiga y dificultad para concentrarse, además de mayor vulnerabilidad a desencadenar depresión (Edgar y McClung, 2013; Germain y Kupfer, 2008). En estos individuos también se ha reportado mayor vulnerabilidad de incurrir en la ingestión de drogas y alcohol (Morikawa et al., 2013). Es difícil discernir la contribución de la luz por la noche sobre la cantidad de alteraciones que presenta el trabajador nocturno, ya que estos individuos además de la contaminación lumínica, están expuestos a privación de sueño y alteraciones circadianas, que por sí mismos son factores que desencadenan muchas de estas alteraciones fisiológicas y de la conducta.

Para lograr deslindar el efecto de la luz nocturna sobre el metabolismo, un estudio realizado con una población de la región de Nara, en Japón, comparó personas que se exponían a bajos o nulos niveles de luz por la noche (menos de 3 lux) con personas que se exponían a niveles mayores de 3 lux (Obayashi et al., 2013). El grupo que se exponía a mayor intensidad de luz presentó una mayor incidencia de obesidad y alteraciones en los niveles de lípidos circulantes, confirmando que la exposición a la luz por la noche desencadena disfunción metabólica.

Un espacio para determinar los efectos nocivos de la luz por la noche, son las unidades de cuidados intensivos en los hospitales, ya que en general éstas se mantienen siempre con la luz prendida para permitir al personal médico y de enfermería una supervisión eficiente de los pacientes. En un estudio realizado en la unidad de terapia intensiva neonatal del Hospital Juárez de México, nuestro grupo demostró que los bebés prematuros internados debido a bajo peso corporal crecen y maduran más lentamente en condiciones de luz constante, en comparación con los bebés prematuros expuestos a un ciclo de luz-oscuridad. Para lograr esto, a algunos bebés se les colocó durante la noche un casco de acrílico con una cubierta de tela que producía penumbra sobre su cabeza y ojos. Comparados con bebés que no recibieron este tratamiento, a las tres semanas 
de estancia en la unidad, los bebés expuestos a la oscuridad por la noche habían ganado 150 g más de peso y el tiempo de estancia hospitalaria se redujo a la mitad, siendo para este grupo un promedio de 30 días para ser dados de alta y para el grupo testigo de 60 días (Vasquez-Ruiz et al., 2014). Otros estudios realizados en diferentes unidades de terapia intensiva han reportado efectos similares, demostrando que la oscuridad por la noche mejora la capacidad de los bebés para asimilar el alimento y por lo mismo logran aumentar de peso para ser dados de alta (Blackburn y Patteson, 1991; Rivkees, Mayes, Jacobs, y Gross, 2004). Estos hallazgos son un claro ejemplo de la importancia que tiene la oscuridad nocturna desde los primeros días de vida.

\section{Los modelos experimentales corroboran los efectos adversos de la luz por la noche sobre la salud}

En años recientes diversos grupos dedicados a la investigación básica han abordado los efectos de la exposición a la luz nocturna sobre la salud con modelos experimentales, principalmente en roedores.

Se sabe que durante la etapa fetal la función circadiana depende de señales maternas, especialmente de la melatonina, la cual es una de las pocas hormonas capaces de atravesar la barrera placentaria (Seron-Ferre et al., 2012). La exposición de la hembra gestante a luz por la noche abole su propia producción de melatonina y repercute en los ritmos circadianos que exhiben sus crías, las cuales presentan bajo crecimiento intrauterino y deficiencia de secreción de corticosterona en etapas postnatales (Mendez et al., 2012).

Canal, Mohammed y Rodriguez (2009) demostraron que la luz por la noche durante el desarrollo daña al reloj biológico de forma permanente, lo que resulta

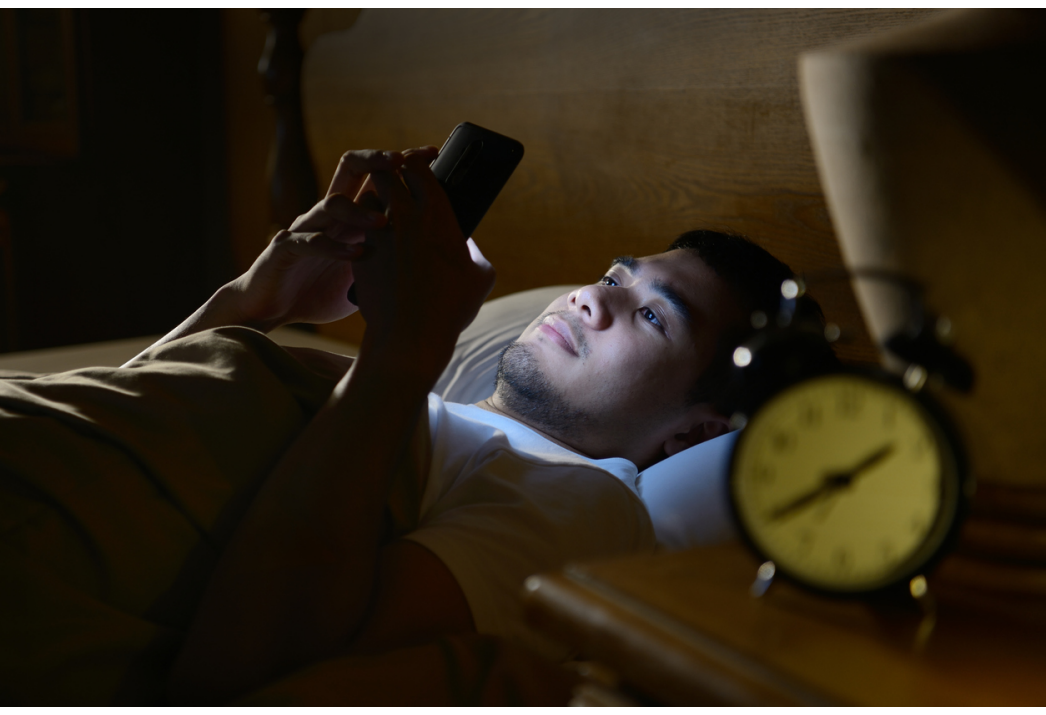
en una menor cantidad de neuronas y células gliales en el NSQ. Las crías de ratón expuestas a luz por la noche durante la lactancia fueron incapaces de exhibir ritmos circadianos coordinados a lo largo de su vida (Ohta, Mitchell y McMahon, 2006). Roman y Karlsson (2013) demostraron que ratas expuestas a ciclos alterados de luz durante la lactancia presentaban durante su etapa adulta conductas de ansiedad, alteraciones en la interacción social y un rendimiento deficiente en pruebas de reconocimiento de formas. Ohta y colaboradores proponen que durante el desarrollo el exceso de luz por la noche altera la organización cerebral y puede ser un factor de riesgo para desarrollar depresión, ansiedad y otras alteraciones del estado de ánimo (2006). 
La luz por la noche también altera la expresión de ritmos circadianos en roedores adultos. A nivel del NSQ, se ha descrito que condiciones de luz tenue por la noche inhiben totalmente la actividad de las células que reciben las proyecciones de la retina y que normalmente mantienen la sincronía entre el ciclo luz-oscuridad y el funcionamiento del NSQ (Isobe y Nishino, 1998). A partir del tercer día después de estar bajo la influencia de la luz por la noche, el NSQ pierde más del 50\% de su actividad rítmica (Coomans et al., 2013); y después de dos semanas en esta condición, también las células que transmiten ritmicidad al resto del organismo muestran baja actividad (Isobe y Nishino, 1998).

En nuestro grupo de investigación encontramos que después de ocho semanas de condiciones de luz por la noche, las ratas adultas perdían el ritmo circadiano de actividad y temperatura corporal. Esto se asoció con niveles muy bajos de activación neuronal en el NSQ y pérdida del ritmo de melatonina. Con una prueba de consumo de azúcar determinamos conductas tipo depresivas. También observamos aumentadas las conductas de ansiedad, lo cual nos llevó a proponer que las ratas bajo condiciones de luz por la noche desarrollan conductas similares a la depresión (Tapia-Osorio et al., 2013). Los niveles altos de ansiedad y depresión asociados con la exposición crónica de luz por la noche son el efecto más consistente reporta-

do en modelos experimentales (Fonken et al., 2009; Fonken, Kitsmiller, Smale y Nelson, 2012; Fonken y Nelson, 2013; Ma et al., 2007; Tapia-Osorio et al., 2013). Además, se ha reportado que la luz por la noche disminuye el rendimiento en pruebas de memoria espacial en ratas (Fujioka et al., 2011).

A mediano plazo (unas cuantas semanas) los animales expuestos a luz tenue (5 lux) por la noche muestran índices de síndrome metabólico y sobrepeso (Fonken, Aubrecht, Melendez-Fernandez, Weil y Nelson, 2013; Fonken y Nelson, 2014). Además, muestran mayor ganancia de masa corporal, mayor acumulación de tejido adiposo visceral (Dauchy et al., 2010; Wideman y Murphy, 2009) y una disminución en la secreción de insulina (Qian, Block, Colwell y Matveyenko, 2013) lo que resulta en un deficiente manejo de glucosa y se parece a un estadio pre-diabético.

Recientemente, nuestro grupo evaluó los efectos de la exposición a luz por la noche sobre el desarrollo de tumores y el sistema inmune. Para explorar lo anterior, se expusieron ratas a luz por la noche (LL) y se compararon con 
ratas sometidas a un ciclo regular de luz-oscuridad (LD) durante cinco semanas. La exposición a LL indujo sobrepeso, elevados niveles de triglicéridos y glucosa en la sangre, así como intolerancia a la glucosa. Cuando las infectamos con un componente de una bacteria, las ratas LL aumentaron los signos de enfermedad (fiebre, pérdida de peso y del apetito) y mostraron elevados indicadores de inflamación. De forma importante, las ratas expuestas a LL desarrollaron tumores de mayor volumen al ser inoculadas con células tumorales. En los tumores extraídos de los animales expuestos a LL, se encontraron aumentados genes involucrados en crecimiento tumoral. Lo anterior sugiere que la exposición a luz por la noche provee un ambiente metabólico propicio en el hospedero el cual favorece el crecimiento de los tumores (Guerrero-Vargas et al., 2017).

Con base en lo anterior, los modelos experimentales han confirmado que la luz por la noche, aún de baja intensidad, promueve perturbaciones fisiológicas y conductuales, aumenta el riesgo de padecer alteraciones en el metabolismo de carbohidratos y lípidos, altera los patrones de descanso e incluso la respuesta inmune, lo cual promueve enfermedades crónicas como ateroesclerosis, cáncer, diabetes tipo 2 y problemas que derivan en síndrome metabólico (Fonken, Weil, \& Nelson, 2013; Zimberg, Fernandes Junior, Crispim, Tufik, \& de Mello, 2012).

\section{Conclusiones y perspectivas}

Los datos experimentales apuntan a que la luz por la noche es un factor que desencadena enfermedades crónicas. Actualmente hemos optado por un estilo de vida similar al del trabajador nocturno, ya que permanecemos despiertos hasta muy entrada la noche y durante ese periodo estamos expuestos a la luz de las computadoras, la televisión o el celular, además de la luz blanca de los focos en casa. La falta de oscuridad promovida por una excesiva exposición a diferentes intensidades de luz provoca alteraciones circadianas y puede inducir diversos problemas de salud. Como hemos discutido, diversos estudios clínicos reportan que la exposición a luz por la noche se asocia con una mayor incidencia de depresión, así como un aumento en el sobrepeso, obesidad e incluso desarrollo de tumores.

La inhibición de la producción de melatonina, las alteraciones circadianas y del sueño son sólo algunos de los posibles mecanismos por los cuales la luz por la noche afecta la fisiología de los individuos y, por lo tanto, promueve el desarrollo de enfermedades (ver figura 3). Los mecanismos exactos que asocian la exposición a la luz por la noche con el desarrollo de enfermedades aún son desconocidos.

Una limitación importante de los estudios con los que se cuenta hasta el momento es que están asociados otros factores que también afectan la salud. Entre ellos la mala calidad de sueño que se asocia al estar despierto y con la luz 
prendida. Para algunos individuos se asocia también con el trabajo nocturno que conlleva actividad y alimentación en horas de dormir.

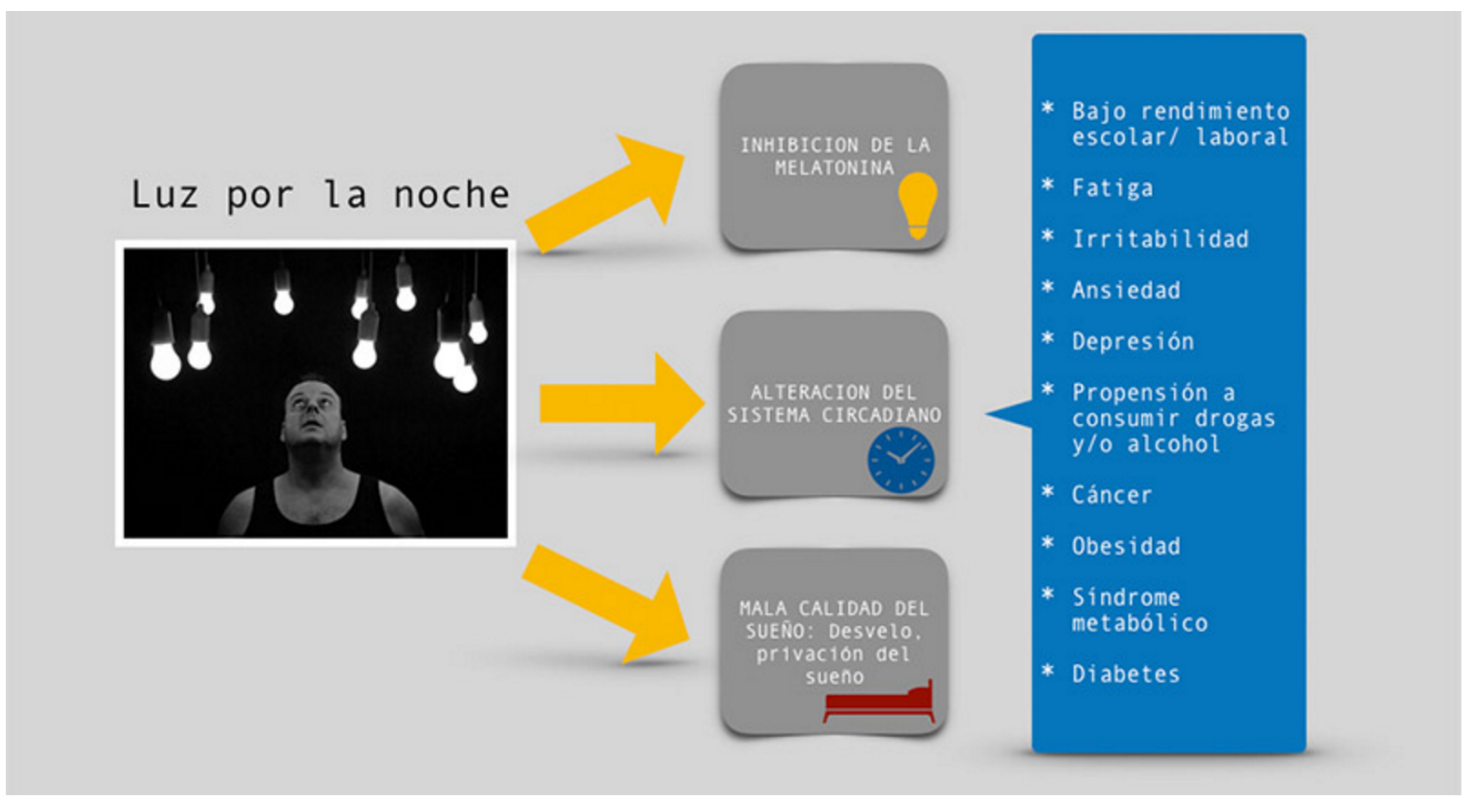

Por otro lado, la limitación en el uso de animales experimentales radica en que, en su mayoría, los roedores son de actividad nocturna. En este sentido y contrario al humano, la exposición a la luz por la noche coincide con su fase de actividad normal. Para poder remediar esta incongruencia, hacen falta estudios con roedores diurnos que semejen mejor las condiciones humanas.

Ante el problema de la exposición a la luz por la noche que enfrenta la sociedad moderna, es necesario contar con más material de divulgación que permita informar a la población sobre el riesgo que representa la contaminación lumínica para la salud y sobre la relevancia de respetar el sueño y descanso por la noche. Respetar la oscuridad por la noche oscureciendo las habitaciones en donde dormimos es una medida simple, que mejorará el descanso y, por lo tanto, el estado de salud física y mental.

\section{Referencias}

* Baez-Ruiz, A., Guerrero-Vargas, N. N., Cazarez-Marquez, F., Sabath, E., Basualdo, M. D. C., Salgado-Delgado, R., ... Buijs, R. M. (2017). Food in synchrony with melatonin and corticosterone relieves constant light disturbed metabolism. J Endocrinol, 235(3), 167-178. DOI: http://doi.org/10.1530/joe-17-0370.

* Blackburn, S., \& Patteson, D. (1991). Effects of cycled light on activity state and cardiorespiratory function in preterm infants. J Perinat Neonatal Nurs, 4(4), 47-54. 
* Boivin, D. B., Duffy, J. F., Kronauer, R. E., \& Czeisler, C. A. (1996). Dose-response relationships for resetting of human circadian clock by light. Nature, 379(6565), 540-542. DOl: http://doi.org/10.1038/379540a0.

* Buijs, R. M., \& Kalsbeek, A. (2001). Hypothalamic integration of central and peripheral clocks. Nat Rev Neurosci, 2(7), 521-526. DOI: http://doi.org/10.1038/35081582.

* Buijs, R. M., van Eden, C. G., Goncharuk, V. D., \& Kalsbeek, A. (2003). The biological clock tunes the organs of the body: timing by hormones and the autonomic nervous system. J Endocrinol, 177(1), 17-26.

* Canal, M. M., Mohammed, N. M., \& Rodriguez, J. J. (2009). Early programming of astrocyte organization in the mouse suprachiasmatic nuclei by light. Chronobiol Int, 26(8), 1545-1558. DOI: http://doi.org/10.3109/07420520903398542.

* Chepesiuk, R. (2009). Missing the dark: health effects of light pollution. Environ Health Perspect, 117(1), A20-27.

* Cho, C. H., Lee, H. J., Yoon, H. K., Kang, S. G., Bok, K. N., Jung, K. Y., ... Lee, E. I. (2016). Exposure to dim artificial light at night increases REM sleep and awakenings in humans. Chronobiol Int, 33(1), 117-123. DOI: http://doi.org/10.3109/07420 528.2015.1108980.

* Coomans, C. P., van den Berg, S. A., Houben, T., van Klinken, J. B., van den Berg, R., Pronk, A. C., ... Meijer, J. H. (2013). Detrimental effects of constant light exposure and high-fat diet on circadian energy metabolism and insulin sensitivity. Faseb j, 27(4), 1721-1732. DOI: http://doi.org/10.1096/fj.12-210898.

* Danilenko, K. V., Cajochen, C., \& Wirz-Justice, A. (2003). Is sleep per se a zeitgeber in humans? J Biol Rhythms, 18(2), 170-178. DOl: http://doi. org/10.1177/0748730403251732.

* Dauchy, R. T., Dauchy, E. M., Tirrell, R. P., Hill, C. R., Davidson, L. K., Greene, M. W., . .. Blask, D. E. (2010). Dark-phase light contamination disrupts circadian rhythms in plasma measures of endocrine physiology and metabolism in rats. Comp Med, 60(5), 348-356.

* Edgar, N., \& McClung, C. A. (2013). Major depressive disorder: a loss of circadian synchrony? Bioessays, 35(11), 940-944. DOI: http://doi.org/10.1002/bies.201300086.

* Escobar, C., Salgado-Delgado, R., Gonzalez-Guerra, E., Tapia Osorio, A., Angeles-Castellanos, M., \& Buijs, R. M. (2011). Circadian disruption leads to loss of homeostasis and disease. Sleep Disord, 2011, 964510. DOI: http://doi. org/10.1155/2011/964510.

* Falchi, F., Cinzano, P., Elvidge, C. D., Keith, D. M., \& Haim, A. (2011). Limiting the impact of light pollution on human health, environment and stellar visibility. J Environ Manage, 92(10), 2714-2722. DOI: http://doi.org/10.1016/j.jenvman.2011.06.029.

* Fonken, L. K., Aubrecht, T. G., Melendez-Fernandez, O. H., Weil, Z. M., \& Nelson, R. J. (2013). Dim light at night disrupts molecular circadian rhythms and increases body weight. J Biol Rhythms, 28(4), 262-271. DOI: http://doi. org/10.1177/0748730413493862. 
* Fonken, L. K., Finy, M. S., Walton, J. C., Weil, Z. M., Workman, J. L., Ross, J., \& Nelson, R. J. (2009). Influence of light at night on murine anxiety- and depressivelike responses. Behav Brain Res, 205(2), 349-354. DOI: http://doi.org/10.1016/j. bbr.2009.07.001.

* Fonken, L. K., Kitsmiller, E., Smale, L., \& Nelson, R. J. (2012). Dim nighttime light impairs cognition and provokes depressive-like responses in a diurnal rodent. J Biol Rhythms, 27(4), 319-327. DOI: http://doi.org/10.1177/0748730412448324.

* Fonken, L. K., \& Nelson, R. J. (2013). Dim light at night increases depressive-like responses in male C3H/HeNHsd mice. Behav Brain Res, 243, 74-78. DOl: http://doi. org/10.1016/j.bbr.2012.12.046.

* Fonken, L. K., \& Nelson, R. J. (2014). The effects of light at night on circadian clocks and metabolism. Endocr Rev, 35(4), 648-670. DOI: http://doi.org/10.1210/er.20131051 10.1210/er.9013-1051.

Fonken, L. K., Weil, Z. M., \& Nelson, R. J. (2013). Mice exposed to dim light at night exaggerate inflammatory responses to lipopolysaccharide. Brain Behav Immun, 34, 159-163. DOI: http://doi.org/10.1016/j.bbi.2013.08.011.

* Fujioka, A., Fujioka, T., Tsuruta, R., Izumi, T., Kasaoka, S., \& Maekawa, T. (2011). Effects of a constant light environment on hippocampal neurogenesis and memory in mice. Neurosci Lett, 488(1), 41-44. DOI: http://doi.org/10.1016/j.neulet.2010.11.001.

* Germain, A., \& Kupfer, D. J. (2008). Circadian rhythm disturbances in depression. Hum Psychopharmacol, 23(7), 571-585. DOI: http://doi.org/10.1002/hup.964.

* Golombek, D. A., \& Rosenstein, R. E. (2010). Physiology of circadian entrainment. Physiol Rev, 90(3), 1063-1102. DOI: http://doi.org/10.1152/physrev.00009.2009.

* Gonciarz, M., Gonciarz, Z., Bielanski, W., Mularczyk, A., Konturek, P. C., Brzozowski, T., \& Konturek, S. J. (2010). The pilot study of 3-month course of melatonin treatment of patients with nonalcoholic steatohepatitis: effect on plasma levels of liver enzymes, lipids and melatonin. J Physiol Pharmacol, 61(6), 705-710.

* Gooley, J. J., Chamberlain, K., Smith, K. A., Khalsa, S. B., Rajaratnam, S. M., Van Reen, E., ... Lockley, S. W. (2011). Exposure to room light before bedtime suppresses melatonin onset and shortens melatonin duration in humans. J Clin Endocrinol Metab, 96(3), E463-472. DOI: http://doi.org/10.1210/jc.2010-2098.

* Grone, B. P., Chang, D., Bourgin, P., Cao, V., Fernald, R. D., Heller, H. C., \& Ruby, N. F. (2011). Acute light exposure suppresses circadian rhythms in clock gene expression. J Biol Rhythms, 26(1), 78-81. DOl: http://doi.org/10.1177/0748730410388404.

* Grundy, A., Sanchez, M., Richardson, H., Tranmer, J., Borugian, M., Graham, C. H., \& Aronson, K. J. (2009). Light intensity exposure, sleep duration, physical activity, and biomarkers of melatonin among rotating shift nurses. Chronobiol Int, 26(7), 1443-1461. DOI: http://doi.org/10.3109/07420520903399987.

* Guerrero-Vargas, N. N., Guzman-Ruiz, M., Fuentes, R., Garcia, J., Salgado-Delgado, R., Basualdo Mdel, C., ... Buijs, R. M. (2015). Shift Work in Rats Results in Increased Inflammatory Response after Lipopolysaccharide Administration: 
A Role for Food Consumption. J Biol Rhythms, 30(4), 318-330. DOI: http://doi. org/10.1177/0748730415586482.

- Guerrero-Vargas, N. N., Navarro-Espindola, R., Guzman-Ruiz, M. A., Basualdo, M. D. C., Espitia-Bautista, E., Lopez-Bago, A., .. . Escobar, C. (2017). Circadian disruption promotes tumor growth by anabolic host metabolism; experimental evidence in a rat model. BMC Cancer, 17(1), 625. DOI: http://doi.org/10.1186/s12885-0173636-3.

* He, C., Anand, S. T., Ebell, M. H., Vena, J. E., \& Robb, S. W. (2015). Circadian disrupting exposures and breast cancer risk: a meta-analysis. Int Arch Occup Environ Health, 88(5), 533-547. DOI: http://doi.org/10.1007/s00420-014-0986-x.

* Ikeda, M., Sagara, M., \& Inoue, S. (2000). Continuous exposure to dim illumination uncouples temporal patterns of sleep, body temperature, locomotion and drinking behavior in the rat. Neurosci Lett, 279(3), 185-189.

* Isobe, Y., \& Nishino, H. (1998). AVP rhythm in the suprachiasmatic nucleus in relation to locomotor activity under constant light. Peptides, 19(5), 827-832.

* Kalsbeek, A., Palm, I. F., La Fleur, S. E., Scheer, F. A., Perreau-Lenz, S., Ruiter, M., ... Buijs, R. M. (2006). SCN outputs and the hypothalamic balance of life. J Biol Rhythms, 21(6), 458-469. DOI: http://doi.org/10.1177/0748730406293854.

* Killick, R., Banks, S., \& Liu, P. Y. (2012). Implications of sleep restriction and recovery on metabolic outcomes. J Clin Endocrinol Metab, 97(11), 3876-3890. DOl: http://doi.org/10.1210/ jc.2012-1845.

* Klerman, E. B., Rimmer, D. W., Dijk, D. J., Kronauer, R. E., Rizzo, J. F., 3rd, \& Czeisler, C. A. (1998). Nonphotic entrainment of the human circadian pacemaker. Am J Physiol, 274(4 Pt 2), R991-996.

Knutsson, A. (2003). Health disorders of shift workers. Occup Med (Lond), 53(2), 103-108.

- Lam, R. W. (2006). Sleep disturbances and depression: a challenge for antidepressants. Int Clin Psychopharmacol, 21 Supp/ 1, S25-29. DOI: http://doi.org/10.1097/01. yic.0000195658.91524.61.

* Leproult, R., \& Van Cauter, E. (2010). Role of sleep and sleep loss in hormonal release and metabolism. Endocr Dev, 17, 11-21. DOI: http://doi.org/10.1159/000262524.

Lewy, A. J., Wehr, T. A., Goodwin, F. K., Newsome, D. A., \& Markey, S. P. (1980). Light suppresses melatonin secretion in humans. Science, 210(4475), 1267-1269.

- Luchetti, F., Canonico, B., Betti, M., Arcangeletti, M., Pilolli, F., Piroddi, M., ... Galli, F. (2010). Melatonin signaling and cell protection function. Faseb j, 24(10), 36033624. DOI: http://doi.org/10.1096/fj.10-154450.

\& Ma, W. P., Cao, J., Tian, M., Cui, M. H., Han, H. L., Yang, Y. X., \& Xu, L. (2007). Exposure to chronic constant light impairs spatial memory and influences long-term depression in rats. Neurosci Res, 59(2), 224-230. DOl: http://doi.org/10.1016/j.neures.2007.06.1474. 
* Mendez, N., Abarzua-Catalan, L., Vilches, N., Galdames, H. A., Spichiger, C., Richter, H. G., . . Torres-Farfan, C. (2012). Timed maternal melatonin treatment reverses circadian disruption of the fetal adrenal clock imposed by exposure to constant light. PLoS One, 7(8), e42713. DOI: http://doi.org/10.1371/journal.pone.0042713.

* Morikawa, Y., Sakurai, M., Nakamura, K., Nagasawa, S. Y., Ishizaki, M., Kido, T., . . . Nakagawa, H. (2013). Correlation between shift-work-related sleep problems and heavy drinking in Japanese male factory workers. Alcohol Alcohol, 48(2), 202-206. DOl: http://doi.org/10.1093/alcalc/ags128.

* Nathan, P. J., Burrows, G. D., \& Norman, T. R. (1999). Melatonin sensitivity to dim white light in affective disorders. Neuropsychopharmacology, 21(3), 408-413. DOI: http://doi.org/10.1016/s0893-133x(99)00018-4.

* Navara, K. J., \& Nelson, R. J. (2007). The dark side of light at night: physiological, epidemiological, and ecological consequences. J Pineal Res, 43(3), 215-224. DOI: http://doi.org/10.1111/j.1600-079X.2007.00473.x.

* Navarro-Alarcon, M., Ruiz-Ojeda, F. J., Blanca-Herrera, R. M., MM, A. S., Acuna-Castroviejo, D., Fernandez-Vazquez, G., \& Agil, A. (2014). Melatonin and metabolic regulation: a review. Food Funct, 5(11), 2806-2832. DOI: http://doi.org/10.1039/c4fo00317a.

* Nir, I. (2003). Melatonin for the treatment of disorders in circadian rhythm and sleep: could it form a basis for medication? Receptors Channels, 9(6), 379-385.

- Obayashi, K., Saeki, K., Iwamoto, J., Okamoto, N., Tomioka, K., Nezu, S., .. Kurumatani, N. (2013). Exposure to light at night, nocturnal urinary melatonin excretion, and obesity/dyslipidemia in the elderly: a cross-sectional analysis of the HEIJO-KYO study. J Clin Endocrinol Metab, 98(1), 337-344. DOl: http://doi.org/10.1210/jc.2012-2874.

Ohta, H., Mitchell, A. C., \& McMahon, D. G. (2006). Constant light disrupts the developing mouse biological clock. Pediatr Res, 60(3), 304-308. DOI: http://doi. org/10.1203/01.pdr.0000233114.18403.66.

- Pandi-Perumal, S. R., Srinivasan, V., Maestroni, G. J., Cardinali, D. P., Poeggeler, B., \& Hardeland, R. (2006). Melatonin: Nature's most versatile biological signal? Febs $j$, 273(13), 2813-2838. DOI: http://doi.org/10.1111/j.1742-4658.2006.05322.x.

* Pauley, S. M. (2004). Lighting for the human circadian clock: recent research indicates that lighting has become a public health issue. Med Hypotheses, 63(4), 588596. DOl: http://doi.org/10.1016/j.mehy.2004.03.020.

* Qian, J., Block, G. D., Colwell, C. S., \& Matveyenko, A. V. (2013). Consequences of exposure to light at night on the pancreatic islet circadian clock and function in rats. Diabetes, 62(10), 3469-3478. DOl: http://doi.org/10.2337/db12-1543.

* Rajaratnam, S. M., \& Arendt, J. (2001). Health in a 24-h society. Lancet, 358(9286), 999-1005. DOl: http://doi.org/10.1016/s0140-6736(01)06108-6.

* Rasmussen, D. D., Boldt, B. M., Wilkinson, C. W., Yellon, S. M., \& Matsumoto, A. M. (1999). Daily melatonin administration at middle age suppresses male rat visceral fat, plasma leptin, and plasma insulin to youthful levels. Endocrinology, 140(2), 1009-1012. DOI: http://doi.org/10.1210/endo.140.2.6674. 
* Reiter, R. (2006). Contaminación lumínica: Supresión del ritmo circadiano de melatonina y sus consecuencias para la salud Cronobiología básica y Clinica (pp. 269289). Madrid: Madrid JA, Rol de Lama A.

* Ribeiro, D. C., Hampton, S. M., Morgan, L., Deacon, S., \& Arendt, J. (1998). Altered postprandial hormone and metabolic responses in a simulated shift work environment. J Endocrinol, 158(3), 305-310.

* Rivkees, S. A., Mayes, L., Jacobs, H., \& Gross, I. (2004). Rest-activity patterns of premature infants are regulated by cycled lighting. Pediatrics, 113(4), 833-839.

* Roman, E., \& Karlsson, O. (2013). Increased anxiety-like behavior but no cognitive impairments in adult rats exposed to constant light conditions during perinatal development. Ups J Med Sci, 118(4), 222-227. DOI: http://doi.org/10.310 9/03009734.2013.821191.

* Salgado-Delgado, R., Angeles-Castellanos, M., Buijs, M. R., \& Escobar, C. (2008). Internal desynchronization in a model of night-work by forced activity in rats. Neuroscience, 154(3), 922-931. DOI: http://doi.org/10.1016/j.neuroscience.2008.03.066.

* Salgado-Delgado, R., Angeles-Castellanos, M., Saderi, N., Buijs, R. M., \& Escobar, C. (2010). Food intake during the normal activity phase prevents obesity and circadian desynchrony in a rat model of night work. Endocrinology, 151(3), 1019-1029. DOl: http://doi.org/10.1210/en.2009-0864.

* Schernhammer, E. S., Feskanich, D., Liang, G., \& Han, J. (2013). Rotating night-shift work and lung cancer risk among female nurses in the United States. Am J Epidemiol, 178(9), 1434-1441. DOl: http://doi.org/10.1093/aje/kwt155.

* Seron-Ferre, M., Mendez, N., Abarzua-Catalan, L., Vilches, N., Valenzuela, F. J., Reynolds, H. E., ... Torres-Farfan, C. (2012). Circadian rhythms in the fetus. Mol Cell Endocrinol, 349(1), 68-75. DOl: http://doi.org/10.1016/j.mce.2011.07.039.

* Shuboni, D., \& Yan, L. (2010). Nighttime dim light exposure alters the responses of the circadian system. Neuroscience, 170(4), 1172-1178. DOI: http://doi.org/10.1016/j. neuroscience.2010.08.009.

* Sigurdardottir, L. G., Valdimarsdottir, U. A., Fall, K., Rider, J. R., Lockley, S. W., Schernhammer, E., \& Mucci, L. A. (2012). Circadian disruption, sleep loss, and prostate cancer risk: a systematic review of epidemiologic studies. Cancer Epidemiol Biomarkers Prev, 21(7), 1002-1011. DOI: http://doi.org/10.1158/1055-9965.Epi-12-0116.

* Spiegel, K., Tasali, E., Leproult, R., Scherberg, N., \& Van Cauter, E. (2011). Twentyfour-hour profiles of acylated and total ghrelin: relationship with glucose levels and impact of time of day and sleep. J Clin Endocrinol Metab, 96(2), 486-493. DOI: http://doi.org/10.1210/jc.2010-1978.

* Stevens, R. G., Brainard, G. C., Blask, D. E., Lockley, S. W., \& Motta, M. E. (2014). Breast cancer and circadian disruption from electric lighting in the modern world. CA Cancer J Clin, 64(3), 207-218. DOI: http://doi.org/10.3322/caac.21218.

* Tapia-Osorio, A., Salgado-Delgado, R., Angeles-Castellanos, M., \& Escobar, C. (2013). Disruption of circadian rhythms due to chronic constant light leads to 
depressive and anxiety-like behaviors in the rat. Behav Brain Res, 252, 1-9. DOI: http://doi.org/10.1016/j.bbr.2013.05.028.

- Turek, F. W. (2007). From circadian rhythms to clock genes in depression. Int Clin Psychopharmacol, 22 Suppl 2, S1-8. DOl: http://doi.org/10.1097/01. yic.0000277956.93777.6a.

* Van Cauter, E., Holmback, U., Knutson, K., Leproult, R., Miller, A., Nedeltcheva, A., . .. Spiegel, K. (2007). Impact of sleep and sleep loss on neuroendocrine and metabolic function. Horm Res, 67 Supp/ 1, 2-9. DOl: http://doi.org/10.1159/000097543.

- Vasquez-Ruiz, S., Maya-Barrios, J. A., Torres-Narvaez, P., Vega-Martinez, B. R., RojasGranados, A., Escobar, C., \& Angeles-Castellanos, M. (2014). A light/dark cycle in the NICU accelerates body weight gain and shortens time to discharge in preterm infants. Early Hum Dev, 90(9), 535-540. DOI: http://doi.org/10.1016/j.earlhumdev.2014.04.015.

* Vinogradova, I. A., Anisimov, V. N., Bukalev, A. V., Semenchenko, A. V., \& Zabezhinski, M. A. (2009). Circadian disruption induced by light-at-night accelerates aging and promotes tumorigenesis in rats. Aging (Albany NY), 1(10), 855-865. DOI: http:// doi.org/10.18632/aging.100092.

* Wideman, C. H., \& Murphy, H. M. (2009). Constant light induces alterations in melatonin levels, food intake, feed efficiency, visceral adiposity, and circadian rhythms in rats. Nutr Neurosci, 12(5), 233-240. DOI: http://doi.org/10.1179/147683009×423436.

- Wolden-Hanson, T., Mitton, D. R., McCants, R. L., Yellon, S. M., Wilkinson, C. W., Matsumoto, A. M., \& Rasmussen, D. D. (2000). Daily melatonin administration to middle-aged male rats suppresses body weight, intraabdominal adiposity, and plasma leptin and insulin independent of food intake and total body fat. Endocrinology, 141(2), 487-497. DOl: http://doi.org/10.1210/endo.141.2.7311.

* Zeitzer, J. M., Dijk, D. J., Kronauer, R., Brown, E., \& Czeisler, C. (2000). Sensitivity of the human circadian pacemaker to nocturnal light: melatonin phase resetting and suppression. J Physiol, 526 Pt 3, 695-702.

* Zimberg, I. Z., Fernandes Junior, S. A., Crispim, C. A., Tufik, S., \& de Mello, M. T. (2012). Metabolic impact of shift work. Work, 41 Suppl 1, 4376-4383. DOI: http:// doi.org/10.3233/wor-2012-0733-4376.

\section{Cómo citar este artículo}

* Guerrero-Vargas, Natalí N., Ángeles-Castellanos, Manuel y Escobar Briones, Carolina (2018). Los efectos adversos de la luz artificial por la noche. Revista Digital Universitaria (RDU). Vol. 19, núm. 3 mayo-junio. DOl: http://doi.org/10.22201/ codeic.16076079e.2018.v19n3.a4. 